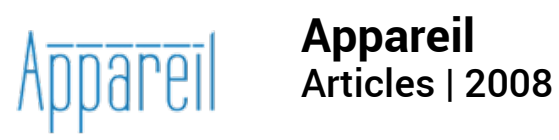

\title{
La spatio-temporalité de la maquette constructiviste : Pourquoi une si longue cohabitation avec le réalisme stalinien?
}

Michel Porchet

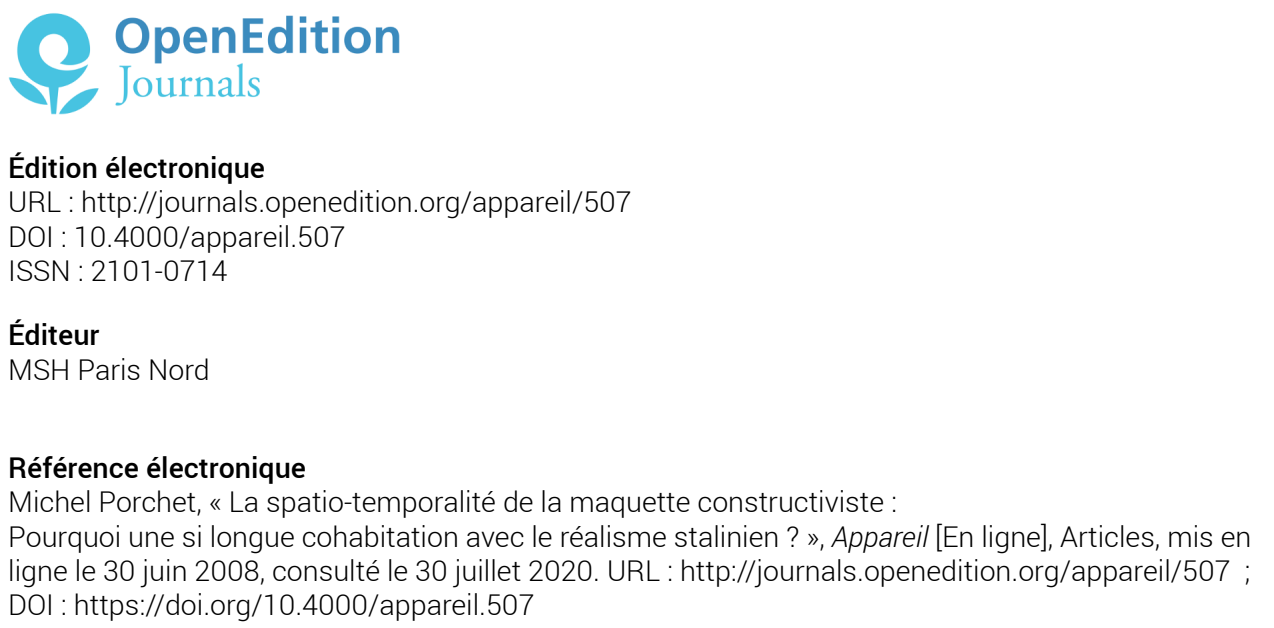

Ce document a été généré automatiquement le 30 juillet 2020.

\section{(c) (1) ()}

Appareil est mis à disposition selon les termes de la Licence Creative Commons Attribution - Pas d'Utilisation Commerciale - Pas de Modification 4.0 International. 


\title{
La spatio-temporalité de la maquette constructiviste : Pourquoi une si longue cohabitation avec le réalisme stalinien?
}

\author{
Michel Porchet
}

\section{La relation à l'industrie et à l'ingénierie}

1 “AVANT-GARDE” : cette métaphore militaire, inventée par Apollinaire, désigne tout mouvement qui «joue ou prétend jouer un rôle précurseur par ses audaces » (Petit Robert). Pour introduire mon sujet je vais simplement montrer quelques œuvres marquantes de l'époque, sans toutefois prétendre qu'elles sont fondatrices, elles illustrent notre propos.

2 Le démon assis (1890) une œuvre presque cubiste de Mikhaïl A. Vroubel, un artiste profondément original et indépendant, peut être aussi important pour les russes que Cézanne en France. Inspiré par l'art byzantin il ordonnance les formes de façon à accentuer la platitude et évacuer la profondeur des tableaux. Il devient le représentant le plus éclatant du style dit "Modern", une version russe de l'Art nouveau. Par le redressement de l'espace sur le plan et le travail de trituration de la couleur, il a fait surgir les composantes matérielles de son tableau, aux dépens de toutes les significations externes, ouvrant ainsi la voie aux expériences radicales du suprématisme et du non-objectivisme. 


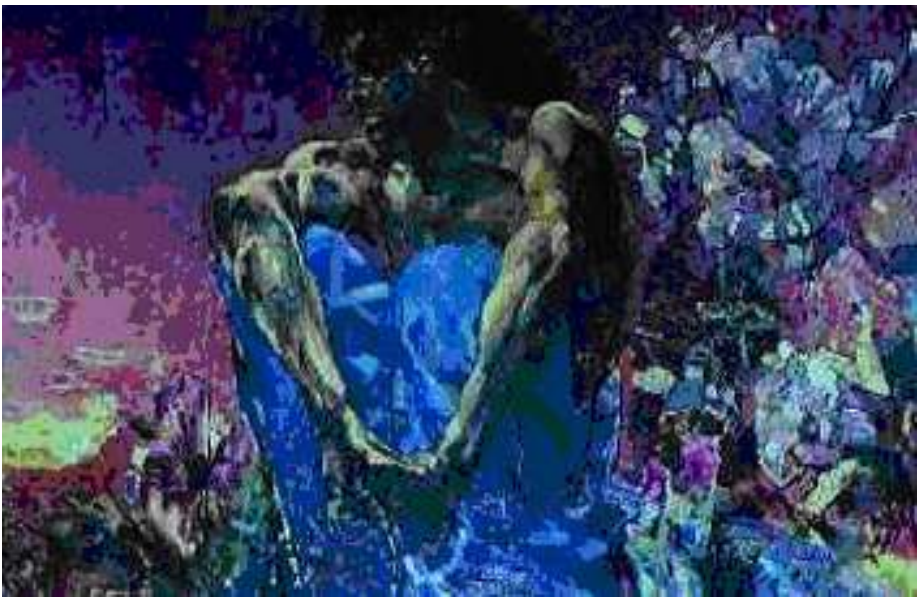

Une oeuvre cubo-futuriste de Malévitch : Tête de paysanne (1912)

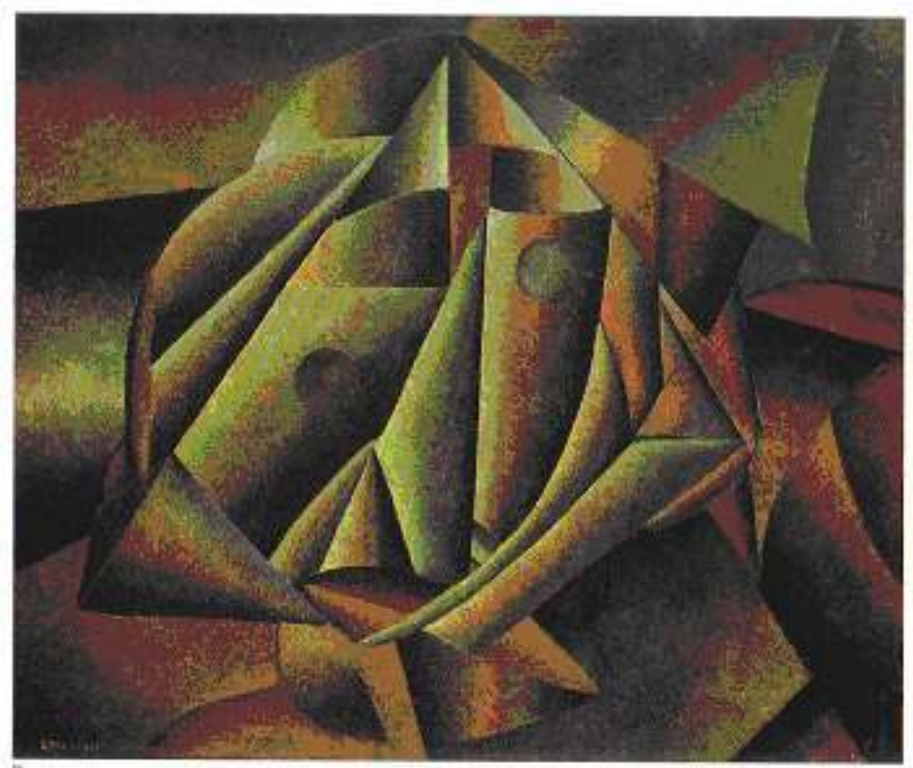

Le Contre Relief de Tatline dont on retrouve une instanciation dans le Café Pittoresque de Georgii Yakulov (Moscou 1917) directement inspiré des rues de Paris qui illustre le côté ludique, souvent oublié des œuvres de l'époque. 

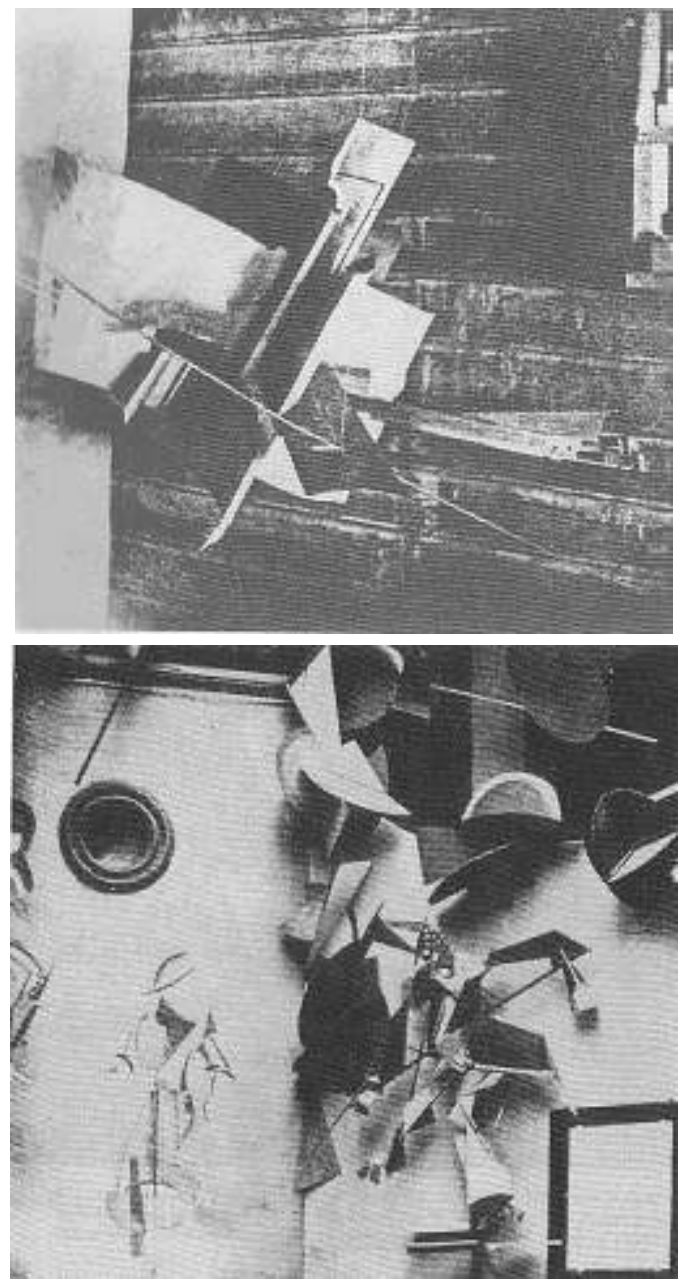

4 Nathan Altman casse, par les lignes avant-gardistes d'une tribune installée à l'occasion du premier anniversaire de la révolution d'Octobre, la verticale majestueuse de la colonne d'Alexandre. En réaction à cette œuvre, le soviet de Petrograd, et il serait trop facile de considérer qu'il est en l'occurrence manipulé par les bolcheviks, exige que le Premier Mai 1919 ne soit pas confié aux futuristes. Cette réaction traduit les relations complexes de l'avant-garde avec le prolétariat révolutionnaire.

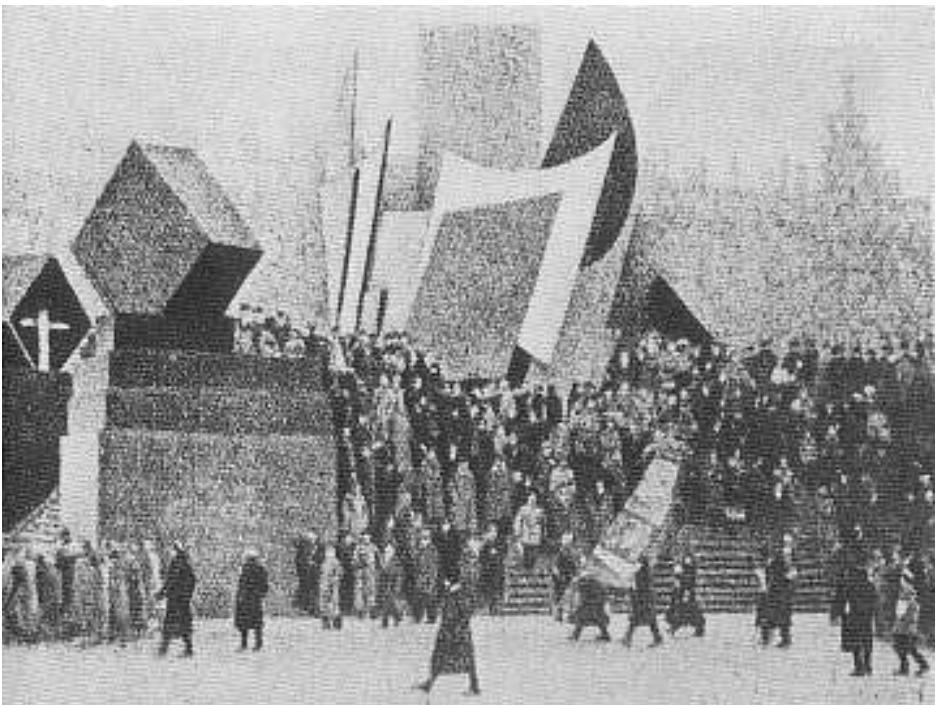


Enfin, l'œuvre de Lissitzki Avec le coin rouge bats les blancs! (1919), fondatrice des pratiques typographiques des constructivistes, s'inscrit, par sa disposition spatiale dans la ligne des Prouns propre à cet artiste.

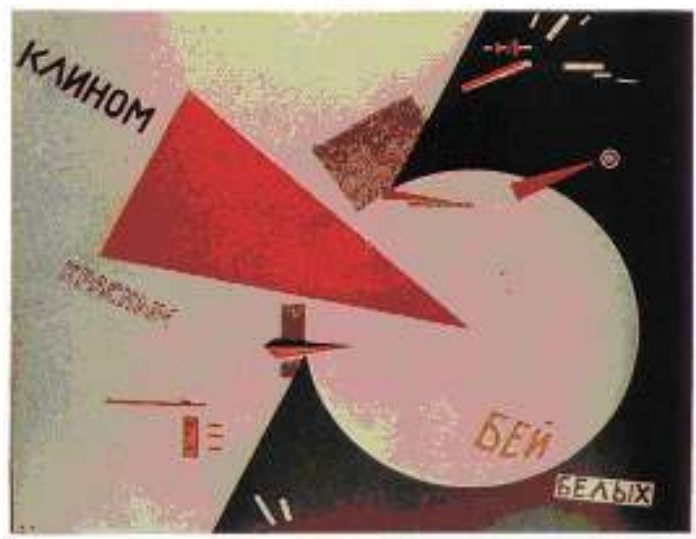

6 Parmi les "Avant-Gardes", parmi les groupes ayant pour intention première de provoquer un changement radical et dont les discours mettent en avant une rupture de principe avec la tradition et avec la pratique et les conventions formelles de leur époque, nous nous limiterons au constructivisme et, au sein de ce mouvement, à Lissitzki. Notre choix aurait aussi pu porter sur Rodchenko qui lui aussi traverse la période qui va de la révolution russe à la deuxième guerre mondiale. Le choix de Lissitzky se justifie par le caractère très international de sa pratique et par les quelques écrits théoriques qu'il a laissé. Son travail artistique va permettre, dans un second temps, de faire une passerelle vers le Bauhaus et de mieux cerner les enjeux du concept d'industries culturelles développé par Adorno.

7 Un manifeste de K.K. Medunetskii et de V. et G. Stenberg dégage les mots clés de la réflexion que je veux conduire :

« Every man born on this earth could, before is return to the soil, go by the shortest route into the factory where the real body of life is fashioned.

This route is called constructivisme the highest springboard for the lead intoi universal human culture. $»^{1}$

8 Le manifeste se poursuit par une condamnation de l'art traditionnel. Les constructivistes mettent en avant un vocabulaire pour l'architecture fondé sur les machines. Ils développent un mode de représentation dans lequel la machine, mais aussi le prolétariat, sont métaphorisés.

9 C'est en partant de l'ingénierie industrielle que je vais tenter de comprendre, avec des clés (des crochets dirait Malévitch) différentes de celles de l'esthétique, certains partis pris de l'Avant-Garde. Les thèses que je vais développer sont les suivantes :

10 - la production industrielle est invoquée mais jamais réellement dévoilée, les objets interviennent en termes de propagande ou sont fétichisés, ils ne sont jamais fonctionnels,

11 - les machines sont figées et vues de l'extérieure, si le mouvement est suggéré par la forme, il est de fait impossible, 


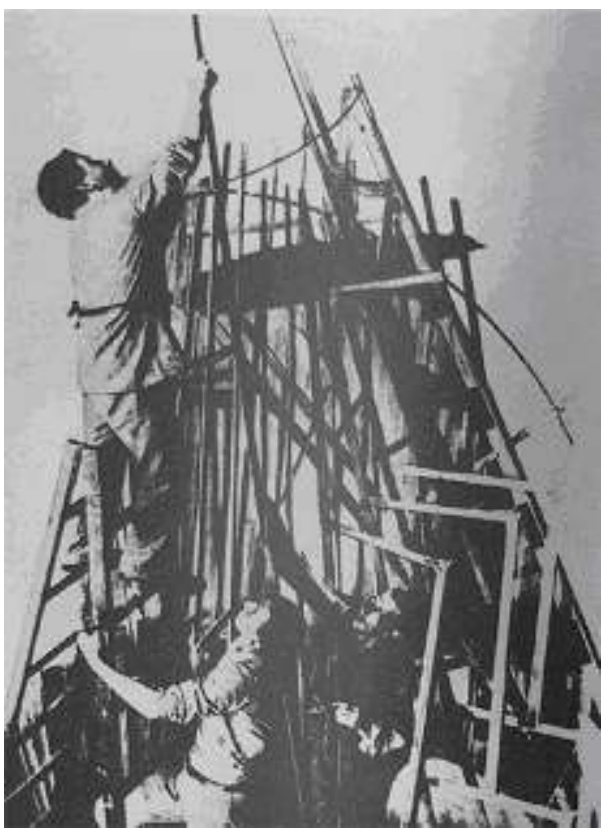

- le rejet de la verticalité correspond à une affirmation du pouvoir de l'homme (ne pas prendre en compte la gravité et la taille), les lois de la physique s'effacent derrière l'enthousiasme productiviste, les KPS²) de GA Stenberg illustrent bien cela ;

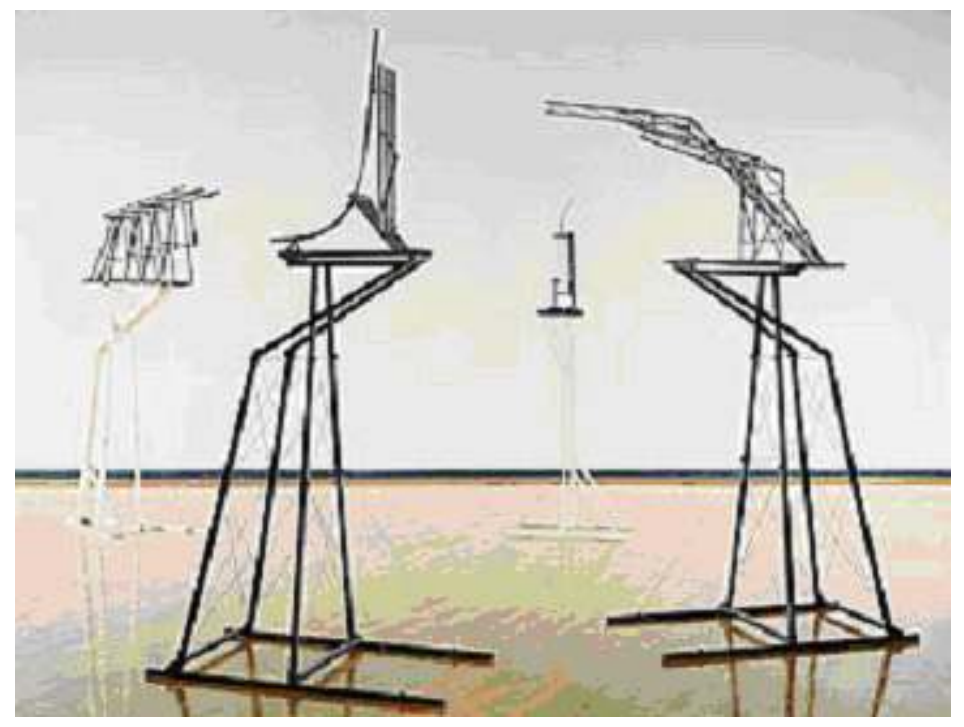

12 - les accointances entre les Avant-Gardes et l'ingénierie industrielle sont beaucoup plus subtiles qu'on ne le pense et souvent fondées sur de profonds malentendus, le rationalisme technique, par un ensemble de mesures pratiquement maîtrisées, parvient à ce que ce qui doit être soit, justifiant ainsi le volontarisme des Avant-Gardes, selon le schéma marxiste, le développement technique résulte d'un développement des forces productives menant à une nécessaire révolution sociale et à la fin du travail aliéné et séparé de la vie, 


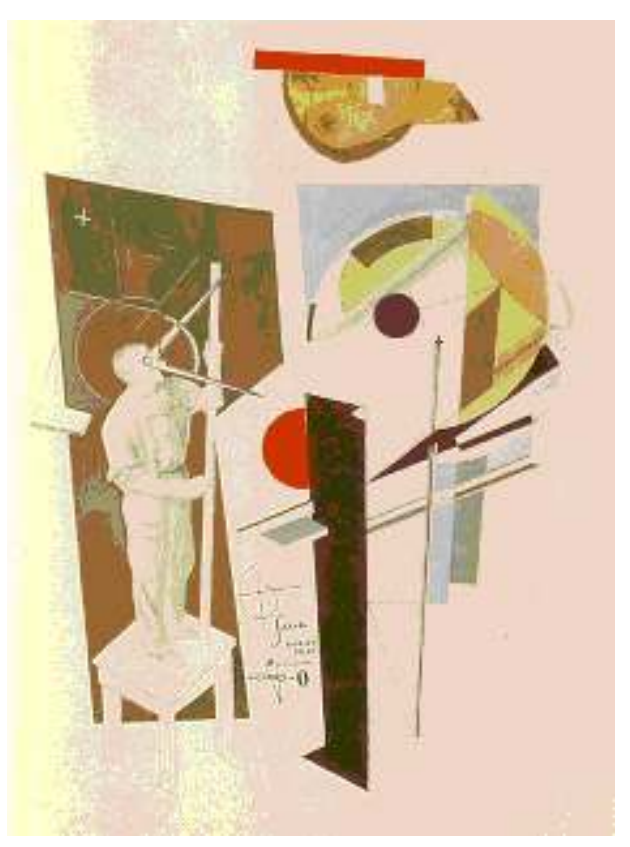

13 - les industries culturelles pointent le nez, si les constructivistes s'intéressent à l'industrie, c'est surtout, en pratique, à la communication industrielle et au design, les lieux de productions médiatiques (presse, radio, cinéma) sont souvent source d'inspiration graphique et/ou objets de projets architecturaux, de nombreux pavillons de foires et expositions sont projetés et quelques fois réalisés, en feuilletant El Lissitzky Jenseits der Abstraktion ${ }^{3}$ on retrouve la plupart des formes qui seront l'objet de l'ire d'Adorno ${ }^{4}$,

14 - l'organisation scientifique du travail de Taylor et l'organisation scientifique de la révolution de Lénine sont parmi les premières applications des méthodes de l'ingénierie aux hommes, la propagande politique est conçue comme une véritable ingénierie sociale, dont le développement traduit un développement des forces productives.

\section{L'industrie mais quelle industrie?}

Depuis le $16^{\mathrm{e}}$ et surtout le $17^{\mathrm{e}}$ siècle, en Europe, on a assisté à une évolution radicale des sciences et des techniques. La coupure entre arts et sciences va diminuer voire disparaître. On assiste à un long processus de formalisation dont le départ peut être daté à $\mathrm{F}$. Bacon et l'aboutissement sur le plan de la science et de la philosophie à Newton et Kant. Il est impossible de dater exactement les origines des sciences et technologies modernes. Selon les cas on soulignera la découverte de l'imprimerie par Gutenberg en 1436, les travaux de Copernic, ceux de Galilée, de Boyle (fondateur du premier laboratoire) ou encore la perspective comme appareil d'une nouvelle forme de la sensibilité.

L'ensemble des activités transformant des ressources naturelles en un produit souhaité forme la production. Du tour de potier jusqu'à l'aube de la révolution industrielle, les transformations des processus pratiques restent mineures. Les arts demeurent une tradition orale propagée du maître au disciple ou par le compagnonnage. La manufacture modifie profondément les rapports sociaux mais ne change que peu les rapports techniques. Aux 17, 18 et $19^{\mathrm{e}}$ siècles, pour ne parler que d'époques 
relativement proches, les concepteurs utilisaient des représentations figuratives pour décrire des machines. Les informations données par les différentes planches suffisaient à la réalisation artisanale d'objets. Dans l'atelier prétaylorien, il y a un contact direct entre le contremaitre responsable de l'atelier et le patron. Le savoir faire de chacun est valorisé. Il est à noter que le changement du rapport intellectuel à la technique que l'on peut faire remonter à la Renaissance précède et anticipe le changement en profondeur des rapports de production.

Une entreprise manufacturière accomplit quatre fonctions majeures (diriger, produire, vendre, assurer les services). Pour la fonction "produire", on retrouve trois fonctions de base (définir produits et procédés / gérer le processus industriel / agir sur le plan physique). La fonction définir regroupe toutes les activités de définition des cahiers des charges ainsi que de conception des produits, des procédés et des équipements. La fonction fabrication transforme les matériaux bruts en produits finis. Elle diffère des autres fonctions de la production car elle manipule (transporte et transforme) des entités physiques. Elle est souvent séparée des autres activités et l'objet d'une organisation propre. La direction de la fabrication est l'unité qui planifie la charge totale du département en ordres de travail, distribue ces ordres sur différents moyens de fabrication, organise la charge de travail pour maintenir efficacité et délais. Quand un ordre de travail est transmis à l'atelier, des ordres de mouvementation sont donnés pour marquer les matières, les outillages et appareils et outils à l'emplacement des machines.

Pour concevoir un objet il faut se le représenter. Réflexion et création exigent un support concret. L'étude traduit des fonctions en surfaces qui sont représentées par des traits. La traduction des fonctions en organes exige un processus complexe de décomposition / synthèse dans lequel la synthèse est souvent déterminante. Malgré les multiples tentatives des théoriciens de la conception, en l'occurrence complices des industriels, pour les briser, les triplets : fonction / forme / matière / conception / fabrication / utilisation résistent et ne se laissent pas découpler facilement. Si l'on insiste pour éclater ces triplets (tentation permanente des théoriciens de la conception), il faut tout de même se rendre à l'évidence : la synthèse est absolument dominante.

Le dessin industriel, fondé sur les notions de cotation et de tolérancement permet de définir les informations nécessaires à la production industrielle d'objets. Le dessin technique n'est pas un modèle de l'objet car il n'est pas la mathématisation d'un monde. Il a, par essence, une double nature il est une représentation ${ }^{5}$ de référence d'une part, et un document, un contrat, fixant les exigences de l'ingénierie d'autre part. Le dessin industriel a ses origines dans des dispositifs que nous avons abordé, sous des éclairages différents, dans le programme $\mathrm{AAD}$ : la géométrie de la figure (Thalès, Euclide), la projection (Papus, Desargues) synthétisée par la géométrie descriptive (Monge) ainsi que le « disegno » et les « théâtres de machines».

Le concepteur mécanicien travaille sur les outils, les machines (de guerre), l'horloge, le véhicule, soit sur le mouvement et le temps court (typiquement la seconde). Un mécanisme, même élémentaire, c'est un ensemble de pièces coopérant dans la réalisation d'une fonction prédéfinie, le plus souvent un mouvement contrôlé. La coopération entre les pièces est assurée par l'intermédiaire de surfaces qui leurs sont idéalement communes. A la lecture d'un dessin, pour comprendre un mécanisme, il faut repérer les surfaces fonctionnelles et analyser la façon dont elles assurent une position 
déterminée entre différentes pièces ou, au contraire le libre débattement qu'elles autorisent. Cette dimension cinématique est dominante dans le déroulement de l'étude. Il y a irruption d'une projection supplémentaire (il serait plus correct de parler d'un repliement) : une représentation purement spatiale d'un mouvement, d'où il résulte une irruption, fantomatique, du temps propre du mécanisme étudié. La relation au temps, du plan, de la figure, du tracé de l'étude de mécanique est donc radicalement différente de celle du plan de l'architecte. La temporalité n'est pas non plus la même, celle de l'ingénieur est clairement celle de la production industrielle. La mort, l'obsolescence de l'objet est inscrite d'emblée dans son programme, stade que n'a pas atteint l'architecture même si certains y voient un horizon vers lequel tendre. Ce temps et cette temporalité de l'industrie n'est pas réellement perçu par les Avants-Gardes qui en arrivent ainsi à percevoir comme émancipatrice, une temporalité qui est celle de l'horloge mécanique, celle du capitalisme.

«Le Capital se fonde sur une théorie de la marchandise et dégage la loi de la valeur. Or au cœur du travail abstrait, il y a le temps de travail. La mesure de ce temps ne peut devenir un universel, que sous le contrôle de l'horloge mécanique. Le régime de la temporalité, au cœur de la réflexion du Marx engagé dans la rédaction du Capital, est ce temps indifférencié de l'horloge mécanique, une temporalité incompatible avec une réflexion esthétique. $»^{6}$

\section{L'ingénierie sociale, née en 1903, s'applique en 1917}

La révolution industrielle déclenchée vers 1850 a entraîné une séparation croissante entre la conception des produits et leur réalisation. L'industrialisation entraîne la naissance de la notion de pièces interchangeables. Cette interchangeabilité des pièces va s'accompagner d'une interchangeabilité des hommes. C'est le développement l'« Organisation Scientifique du Travail » (OST). Fondé sur les principes posés par F.W. Taylor en 1903, le taylorisme vise à obtenir la productivité maximum par l'intermédiaire d'un bureau des méthodes, qui précise quand et comment le travail doit être exécuté, qui organise les opérations élémentaires nécessaires à l'exécution de ce travail de façon à prévoir, grâce aux données accumulées, les délais d'exécution correspondants, et enfin qui prépare les instructions concernant les modalités d'exécution et l'outillage nécessaire. Les pivots du système reposent sur l'analyse des mouvements de l'ouvrier et le chronométrage. Ce mode d'organisation entraîne une sous exploitation du potentiel humain, car en regroupant des machines similaires les pièces vont de l'une à l'autre de façon aléatoire dans des implantations faites sans rapport avec les processus de fabrication. Il en résulte une très grande complexité de la mouvementation en particulier et des difficultés de gestion qui ne se résolvent que par l'allongement des délais. Dans les années 10-20, H. Ford proposera une organisation, taylorienne dans son principe, mais orientée vers la grande série. Les postes de travail et machines sont reliés par une chaîne synchronisant les activités des machines mais aussi des hommes.

Lénine est un grand admirateur du système de Taylor, mais il n'est pas le seul. Arvatov, théoricien du productivisme, affirme que :

«Les tendances de la grande production de notre temps, et donc de l'industrie soviétique nationalisée aussi, en passant par l'organisation scientifique du travail (naucnaja organizacija truda (NOT)) ou taylorisme, n'aboutissent pas à une offre spontanée du marché, mais à l'organisation directe de la consommation par l'industrie centralisée. Elles mènent au calcul collectivisé de masse des besoins de la 
société, à leur satisfaction rationnelle donc à une inventivité productive et planifiée. $»^{7}$

Si 1903 est l'année d'apparition du taylorisme c'est aussi l'année de parution du Que Faire? de Lénine, manuel décrivant la méthode scientifique de prise du pouvoir par le parti du prolétariat. Ce petit livre est certainement le texte le plus important, dans ses conséquences pratiques, de Lénine. Il servira pendant prêt de trois quarts de siècle à délimiter des partages souvent violents au sein de la gauche révolutionnaire. Que Faire? est un véritable manuel technique de la prise du pouvoir, l'admiration de Lénine pour le système de Taylor n'a rien d'anecdotique, le parti, auto-proclamé avant-garde consciente du prolétariat, est en fait le véritable bureau des méthodes de la révolution.

La tragédie des Avant-Gardes, qu'illustre le destin de Maïakovsky, est d'avoir, dans un prolongement pervers des lumières, perçu comme émancipatrice cette ingénierie nouvelle. Un rapport ambigu s'est formé, petit à petit, entre les artistes de l'AvantGarde et la révolution. La lutte contre le passé uni ces deux éléments, mais la victoire d'Octobre a détruit ce lieu commun. Un état conflictuel s'ouvre entre l'intelligencia et le pouvoir soviétique.

5 La revue Iskousstvo Kommouny (L'art de la Commune), $\left(\mathrm{n}^{\circ} 1,1918\right)$ proclamait :

«Abattre, détruire, faire disparaître de la surface du globe les formes artistiques anciennes - voilà le rêve de tout nouvel artiste, de tout artiste prolétarien, de tout homme nouveau!»

Dans leur volonté destructrice, futuristes et bolcheviks se retrouvaient au coude à coude, frères d'armes dans un seul et même combat. Les uns comme les autres souhaitaient ériger sur les ruines du passé la «Grande Utopie ». Mais alors que les artistes, tel Kandinski, avaient dans l'idée de créer un " édifice universel des arts » les bolcheviks, eux, prévoyaient d'installer tous les êtres humains ayant survécu à l'embrasement révolutionnaire, dans une «maison commune» semblable à celle imaginée par Campanella dans son ouvrage La Cité $d u$ Soleil ${ }^{8}$. Si les futuristes exigent une totale "séparation de l'art et de l'Etat " et une complète liberté de création, les bolcheviks, d'entrée de jeu, misent sur l'« immense rôle éducatif » de l'art. Sur ce point, précisément, leurs voies respectives n'allaient pas tarder à diverger, mais en 1918, les bolcheviks ont encore besoin de l'art pour démontrer au prolétariat qu'il était possible de transformer leurs masures misérables en magnifiques palais pour le peuple.

En 1918, Petrograd, berceau de la Révolution, offre un tableau sinistres. Les rues obscures sont dangereuses pour les passants : vols et agressions, dus à la famine et favorisés par l'absence de tout contrôle se multiplient. La foule russe, exaspérée, trouve un exutoire aux épreuves de la vie quotidienne en pillant les magasins de boissons alcoolisées, en ravageant les appartements des familles riches et en se déchaînant sur le premier venu. La terreur introduite après la tentative d'assassinat contre Lénine n'arrange rien. Pourtant, l'organisation de futures fêtes de masse est à l'ordre du jour chez les bolcheviks. Le premier mai 1918 offre une occasion de "démocratiser l'art ", de le faire sortir des salons et des musées à la rencontre d'un nouveau public. Dès le printemps, les artistes engagés prennent possession des rues et des places. Des principes artistiques souvent opposés sont combinés au service d'un même but. Toute la passion et l'audace débridée de l'art nouveau s'étalent sur d'immenses pièces de tissus presque aussi grandes que les maisons. Des panneaux cubistes, futuristes, ... dissimulent l'architecture impériale. Des lignes abruptes, des taches provocantes rayent avec force les dignes assemblages de pierres des palais, dans une affirmation de 
conceptions esthétiques radicales. A côté de ces novations, on peut rencontrer dans les rues voisines des décorations mêlant guirlandes de verdure, lampions électriques, drapeaux rouges, calicots couverts de slogans, travaux d'amateurs empreints d'une authenticité naïve. Beaucoup de ces décorations murales dénotent un attachement aux traditions simples du passé. Les slogans révolutionnaires sont peints avec les mêmes caractères que ceux d'anciennes revues petites-bourgeoises et encadrés de motifs fleuris. Au-dessus de l'entrée des bâtiments officiels, des oriflammes, dont la composition rappelle celle des blasons des grandes familles, portent l'inscription «le pouvoir aux soviets".

Cette première tentative fut la répétition générale de l'anniversaire d'Octobre. En novembre 1918, Petrograd se transforme pour un temps en une immense scène. Tous les spectacles sont gratuits. Pour la première fois, les ouvriers peuvent pénétrer dans les grands théâtres de la ville. Le théâtre national Marinski présente Boris Godounov avec Chaliapine. Le théâtre de la Perspective Liteiny donne Les Bas-Fonds de Gorki. Dans les locaux du Drame musical, Meyerhold met en scène Misteria-Bouffe de Maïakovski, dans des décors de Malévitch. Le succès le plus grand est remporté par le drame de Romain Rolland, Le Quatorze-Juillet, mis en scène au théâtre du Proletkul't, sous le titre de La Prise de la Bastille. L'œuvre tient à la fois du meeting et de la manifestation, seules formes rituelles propres à la récente culture festive du prolétariat urbain. Enfin, le choix des acteurs, pour la plupart simples ouvriers d'usine, encourage la foule à mêler le théâtre et la vie.

En 1918, divers groupes d'artistes professionnels, enthousiasmés par l'ampleur de la Révolution, s'efforcent de créer un milieu culturel utopique, mais refusent de se dissoudre dans une identité qui les assimile à la masse. Persuadée d'être le centre de l'univers, les artistes, principalement les futuristes, adversaires du folklore et des traditions, ne supportent pas les arches de bois, les guirlandes, les lampions électriques ou simplement colorés qui rappellent les fêtes de l'époque tsariste et tout ce qui, par le passé, a été l'œuvre de la classe bourgeoise du monde entier.

Lénine refuse l'idée de culture prolétarienne. Il faut non pas créer une nouvelle culture mais utiliser le patrimoine transmis par la bourgeoisie et impliquer les intellectuels et les techniciens bourgeois dans l'édification de l'État socialiste. Il soutient le contrôle prolétarien sur la culture, un contrôle effectué par le parti bolchevique. Il est depuis une bonne dizaine d'années en conflit avec Bogdanov', idéologue du Proletkul't, une organisation artistique et littéraire qui se propose de créer une culture faite par et pour le prolétariat. Constituée à la veille de la révolution d'Octobre, le Proletkul't connaît un grand essor de 1917 à 1920. En décembre 1920, une lettre du Comité central du parti communiste russe (bolchevik) sur le Proletkul't met fin au conflit entre le parti et le mouvement pour la culture prolétarienne, en soumettant définitivement ce dernier au Narkompros (Commissariat du peuple à l'Instruction publique). Lénine, plutôt conservateur sur le plan culturel, s'oppose aussi au futurisme comme l'atteste une lettre envoyée à M. N. Pokrovskij en 1921 : « Camarade Pokrovskij, Je vous demande encore et encore de nous aider dans la lutte contre le futurisme $»^{10}$.

31 Le commissaire du peuple à l'Instruction publique, Anatoli Vassiliévitch Lounatcharski ${ }^{11}$ nommé à l'unanimité par les soviets, en octobre 1917 à la tête du Narkompros, contribuera au sauvetage du patrimoine artistique russe. Sa tolérance, en particulier vis à vis du futurisme favorisera l'émergence des Avant-Gardes puis, dans un second temps leur conservera une certaine marge de manœuvre face au pouvoir. 
32 A partir de 1925, la politique du parti à l'égard de l'art change, le climat artistique et littéraire est en pleine mutation, ce qui se traduit dès l'adoption du premier plan quinquennal en 1928. La résolution du CC du PCR(b) du premier juillet 1925, rédigée par Boukharine, semble accorder une large place aux différents courants, mais c'est à cette résolution que firent appel toutes les associations à tendances réalistes pour affirmer leur prédominance. La résolution sur la refonte des organisations littéraires et culturelles de 1932 qui décrète le réalisme socialiste comme la seule forme d'art admise par le régime. Le simulacre s'impose à tous.

33 L'art de la maquette développé par les constructivistes survivra à la condamnation des avant-gardes par le pouvoir soviétique et coexistera avec le réalisme socialiste de l'art officiel. Le numéro Livre de la revue l'URSS en construction dû à Rodtchenko date de 1935, Lissitzky travaillera à cette revue jusqu'à sa mort en 1941.

\section{Les Avant-Gardes russes, l'usine, le travail, le travailleur}

Ce n'est pas ici le lieu pour aborder l'ensemble des mouvements des Avant-Gardes russes. Chagall a rompu tôt et violemment, il n'est pas systématiquement lié à l'avantgarde. Pour Malévitch, le malentendu avec la révolution bolchevique est profond, il suffit de lire ses écrits théoriques pour se convaincre que son œuvre n'a rien à voir avec un quelconque technicisme ou l'un ou l'autre ouvriérisme. Le cas de Kandinsky est plus complexe. Comme je l'ai indiqué lors d'un précédent séminaire ${ }^{12}$ les sources de son inspiration étaient \{Wagner / la perte de l'intégrité de l'atome / la peinture pure de Monet\}. Les rôles de Kandinsky à l'INKHUK (institut culture artistique qui recherche les constituants irréductibles de l'art et une théorie de l'art à l'époque communiste) puis au Bauhaus, ainsi que ses écrits théoriques méritent à eux seuls une étude. Dès 1920, au sein même de l'INHUK, dont il est le fondateur, surgit un débat serré entre Kandinsky et certains membres qui, petit à petit, vont adopter des positions productivistes. Il quitte l'INKHUK, après le rejet de ses propositions et la victoire du productivisme. L'INKHUK se dissoudra dans la VHUTEMAS (1920) (Ateliers supérieurs technicoartistiques d'État) dont la vocation est de créer des artistes particulièrement qualifiés pour l'industrie. Il devient en 28 le VHUTEINI (haute école d'art et institut technique d'Etat). Cette version soviétique du Bauhaus ferme en 1930.

35 C'est à l'intérieur du Front gauche de l'art (LEF, Levyj front iskusstva), né à Moscou en 1922, que le productivisme rencontre le futurisme. Le LEF réunit les théoriciens de l'art comme production (Brik, Arvatov), les partisans de l'art pour la construction de la vie (Koujak) et l'un des premiers théoriciens de la littérature factuelle (Tretiakov). On y retrouve aussi les formalistes (Tynjanov et Sklovskij), les cubo-futuristes (Kamenskij), les constructivistes (Lavinsky, Rodtchenko, Stepanova et Tatline) et enfin, les anciens membres du groupe futuriste Centrifuge (Asséiev et Pasternak). On retrouve aussi des metteurs en scène (Eisenstein, Vertov) et enfin Maïakovsky, dont le passé de futuriste va se fondre avec la recherche qu'il conduit alors sur le nouveau rôle de l'art comme travail, c'est-à-dire comme production :

«Pour la première fois un mot nouveau dans le domaine de l'art - constructivisme

- est venu de Russie et non de France ; on en arrive à s'étonner de trouver ce terme

dans le vocabulaire français. Pas le constructivisme d'artistes qui fabriquent

d'inutiles constructions de tôles et de fil de fer (...), mais le constructivisme qui ne 
conçoit le travail formel que comme une ingénierie destinée à fonder notre vie pratique. $»^{13}$ présente bien des affinités avec le Proletkul't et la pensée de Bogdanov sauf qu'Arvatov, se rapprochant sur ce point de l'orthodoxie bolchevique, reconnaît un sujet historique différent dans sa tentative de récupération de l'ancienne classe des intellectuels au service de son projet de création d'une culture et d'un art prolétariens. L'idéologie productiviste se présente comme une tentative pour supprimer l'art et le transformer en travail. Les galeries, ces lieux surannés destinés à l'exposition d'œuvres mortes doivent disparaitre et l'art faire irruption dans les rues, sur les places et dans la vie de tous les jours. L'art entre dans les usines, là ou l'artiste se dépouille de sa blouse de peintre pour travailler avec les ouvriers, côte à côte, ne prenant plus appui sur des notions obsolètes comme inspiration et fantaisie puisque la créativité participe du processus productif. Les bases du productivisme sont :

1. l'idée de l'ouvrier qui réunit en lui les figures de l'organisateur et de l'exécuteur dans le cadre d'un taylorisme débarrassé de la division du travail,

2. le principe du travail considéré comme un processus créatif et positif

(d'où la formule " production de biens matériels = production de biens artistiques »),

40 3. la conception du travail comme formateur de la vie quotidienne (byt $\left.{ }^{14}\right)$, selon la prémisse que toute l'existence de l'ouvrier est déterminée par sa vie dans l'usine,

41 4. l'exaltation de la machine en tant que collaboratrice de l'homme et porteuse de la nouvelle beauté de la technique.

L'entrée de l'artiste dans la production est avancée comme une réponse au problème de l'industrialisation et de la non-compétitivité de l'industrie soviétique sur le plan international. Pour Arvatov, la production soviétique doit sortir des schémas rigides qui enlèvent toute inventivité et pour ce faire il faut encore avoir recours, dans une première phase, à l'esprit artistique. L'ingénieur organisateur, précurseur de l'artisteingénieur, est une figure tayloriste. Les cadres ingénieurs-constructeurs doivent connaître les méthodes scientifiques et soutenir la comparaison avec les standards américains et avec toute l'industrie moderne. La science, la technique industrielle et l'art fusionnent grâce au travail que conduisent en commun deux figures qui n'ont jamais opéré à l'unisson : l'artiste et l'ingénieur.

43 Le productivisme aspire à un monde dans lequel toutes les frontières entre l'art, le travail et la vie seraient anéanties, et où la vie deviendrait l'œuvre d'art par excellence. Taraboukine proclame que la "source de l'art productiviste se situe dans ce que la vie a de plus vigoureux et non sur le Parnasse », pour un productiviste ce que la vie a de plus vigoureux. c'est précisément le travail. Cette fusion de l'art avec la vie de tous les jours, dans un monde totalement artistique, n'est possible que comme l'art d'un prolétariat économiquement gagnant, comme l'art d'une production collectivisée. 

pisme", ou, plus exactement, de son "conservatisme révolutionnaire", n'est pas une logique théorique, construite et basée sur l'observation de causes et d'effets, mais une logique et un langage métaphoriques, poétiques, imagés. Face à une réalité socio-économique et politique chaotique, face à la crise de la société et de la cultures allemandes, Jünger veut maîtriser les effets pervers, les dysfonctionnements par l'esthétique. Steil conclut : «Le livre [=Le Travailleur] veut éduquer [les hommes] à avoir une attitude souveraine face aux processus sociaux». L'observation minutieuse, froide, dépassionnée, constituerait dont la "clef magique" qui permettrait à l'élite qui s'en sert de maîtriser les crises, de mettre un terme au chaos et aux disparités dissolvantes qui entravent le bon fonctionnement des sociétés qui les subissent. Steil constate le hiatus entre cette vision du "Travailleur » et celle, marxiste et empirique, du « Prolétaire » : la figure forgée par Jünger se place très haut au-dessus des contingences socioéconomiques ; le prolétaire conscient de sa déréliction, lui, travaille au cœur même de ces contingences, sans prendre aucune distance, sans détachement. Les figures du «Travailleur » sont des figures imaginaires, idéalisées à outrance, dés-individualisées et apurées : elles fonctionnent comme des soldats prussiens de l'ère frédéricienne à l'exercice.

La proposition de transformer, par le bolchevisme et le taylorisme, le travail du salarié en un travail créatif et libre, ouvrant à la socialisation du travail artistique et à un monde sans marché, où le producteur et le consommateur ne font qu'un, l'ouvrier devient artiste et l'artiste ouvrier, l'art devenant à la fois production et travail fait du prolétaire un personnage qui n'est pas moins mythique que le « Travailleur » de Jünger. 
En dehors de la question décisive du point de vue politique de la propriété sociale des moyens de production, le travailleur, lui aussi imaginaire, idéalisée à outrance, désindividualisé et apuré des productivistes russes, à la fois produit et source de l'ingénierie, devient figure ultime de la raison dans une société sans division entre le travail manuel et le travail intellectuel.

\section{Le constructivisme par delà l'idéologie un art authentique}

Parmi les artistes constructivistes, Lissitzky est celui dont l'œuvre théorique est la plus consistante. Je citerai simplement ici quelques contributions en relation directe avec notre propos. Claude Leclanche-Boulé n'est pas loin de nier toute ambiguïté du constructivisme :

«"L'art dans la vie", pour reprendre le slogan de Tatline, n'est pas à l'origine la suppression totalitaire de l'art réclamée par les fonctionnalistes, pas plus que sa subordination à la vie quotidienne ou sa soumission à de prétendues fins utilitaires, économiques ou socio-politiques. C'est un projet nettement plus subversif de transformation de la société, hérité du futurisme et développé par les constructivistes, qui passe par la promotion de la créativité et de l'invention au rang des valeurs esthétiques suprêmes; son enjeu principal est certes de nature politique puisqu'il s'agit in fine d'étendre ces modes de création à l'ensemble de la vie collective et individuelle, mais il se situe aux antipodes d'un système politique que les constructivistes avaient identifié à tort avec leur idéologie depuis la Révolution d'Octobre. $»^{17}$

Une telle affirmation demeure un peu facile, si le projet constructiviste n'est pas purement et simplement le projet bolchevique, les croisements entre eux demeurent multiples. Si l'art est dans la vie (du travailleur) il ne lui est pas subordonné, on n'est pas enfermé dans l'utilitarisme, et c'est là que les artistes constructivistes se distinguent des théoriciens du productivisme. Mais l'ambiguïté demeure. Dans Notre livre (URSS), traduit de l'allemand, par C. Leclanche-Boulé à partir du texte publié dans la revue Gutenberg-Jahrbuch 1926, 27, Lissitziky montre sa clairvoyance :

«Chaque invention en art est un événement unique ; elle n'évolue pas. On voit avec le temps se développer autour d'elle des variations sur le même thème, parfois sur un ton plus aigu, parfois sur un ton plus grave, mais on y retrouve rarement la force élémentaire originelle. Les choses vont ainsi jusqu'à ce que l'effet de l'œuvre d'art devienne, par suite d'une longue habitude, si mécaniquement automatique, que l'esprit cesse de réagir à ses moyens épuisés. Alors le temps est venu pour une nouvelle invention."

[...]

«Ils ont la vue courte ceux qui pensent que seule la machine, c'est-à-dire le remplacement des procédures manuelles par des procédures mécaniques, peut apporter des modifications fondamentales dans la forme et l'aspect des choses. C'est le consommateur avec ses exigences propres qui se trouve au premier rang de ceux qui décident des changements; je veux parler bien sûr de la couche sociale d'où surgit la "commande". Aujourd'hui ce n'est plus un cercle étroit, une mince couche superficielle de la société, mais "tous" les individus, la masse. De nos jours, l'idée qui soulève les masses c'est le matérialisme, mais ce qui caractérise notre époque, c'est la dématérialisation. Un exemple : les échanges de correspondance se développent, le volume des lettres, du papier écrit, du matériel utilisé se met à gonfler; alors le téléphone vient soulager cette tension. Puis c'est au réseau électrique, aux installations électriques de s'accroître, et la radio vient les soulager. 
Le matériel se réduit, nous dématérialisons, nous remplaçons des masses encombrantes de matériel en libérant les énergies. C'est le signe de notre époque. »

51 Mais on peut penser qu'il y a des limites à cette clairvoyance. Cette belle citation change quelque peu de portée si on la confronte à l'image suivante :

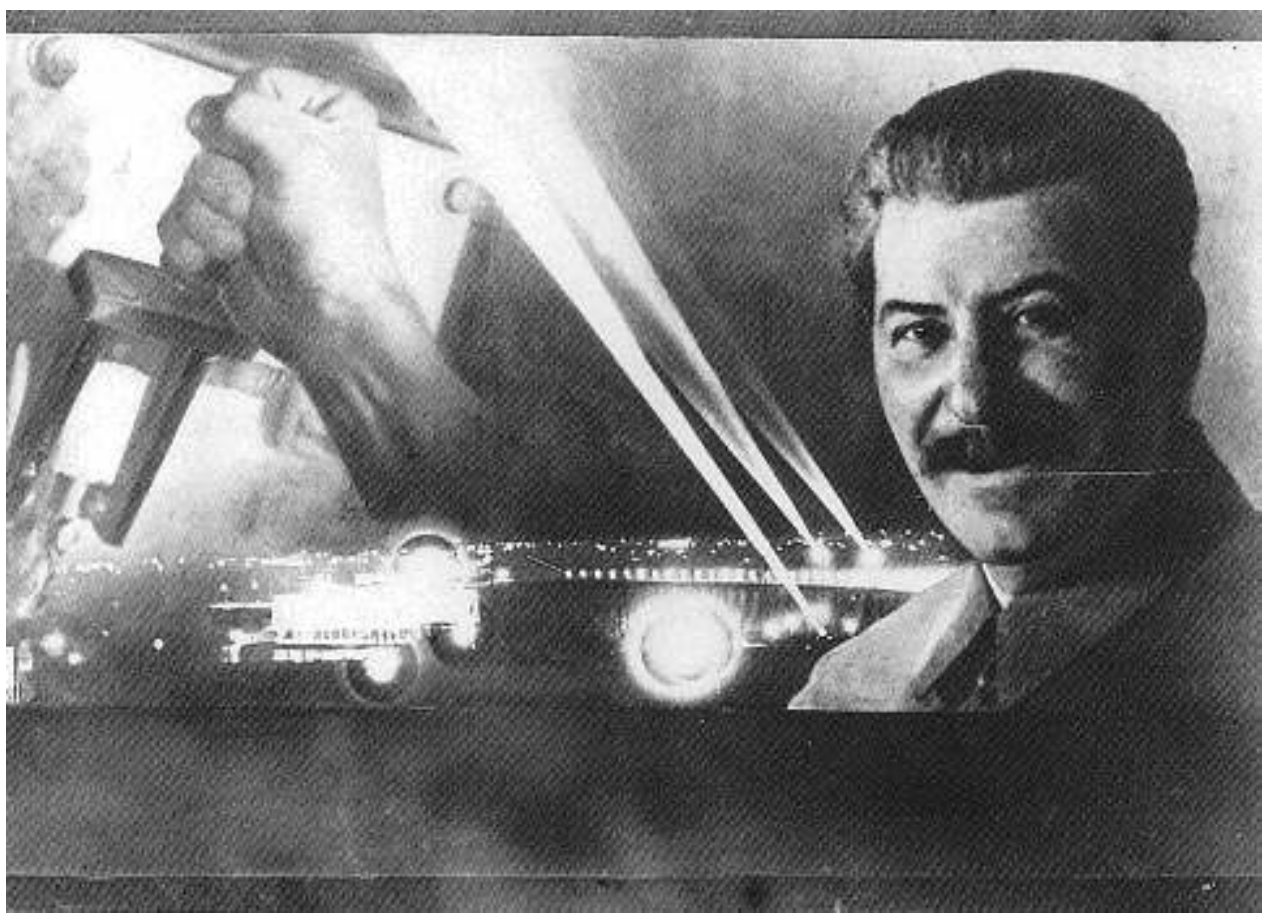

52 Lissitzky évoque ensuite l'intérêt des écritures idéographiques, Marinetti et le mouvement futuriste, ainsi que différents artistes que nous avons déjà évoqués, puis, évoquant des masses russes à demi-illettrées :

«Le livre traditionnel a été déchiré en pages séparées, cent fois agrandies, renforcées par la couleur et exposées dans la rue sous forme d'affiches. Contrairement à l'affiche américaine, notre affiche n'était pas destinée à être saisie au vol et d'un seul coup à partir d'une automobile roulant à toute vitesse, mais à être lue et comprise à courte distance. "

53 Il faut ajouter que les cloisons entre les différents mouvements de l'Avant-Garde sont loin d'être étanches. Dans l'article Réclame paru en 1924 à Bâle, cité lui aussi par C. Leclanche-Boulé il se laisse aller à un certain fonctionnalisme qui transparaît également dans la forme. 


\section{La réclame}

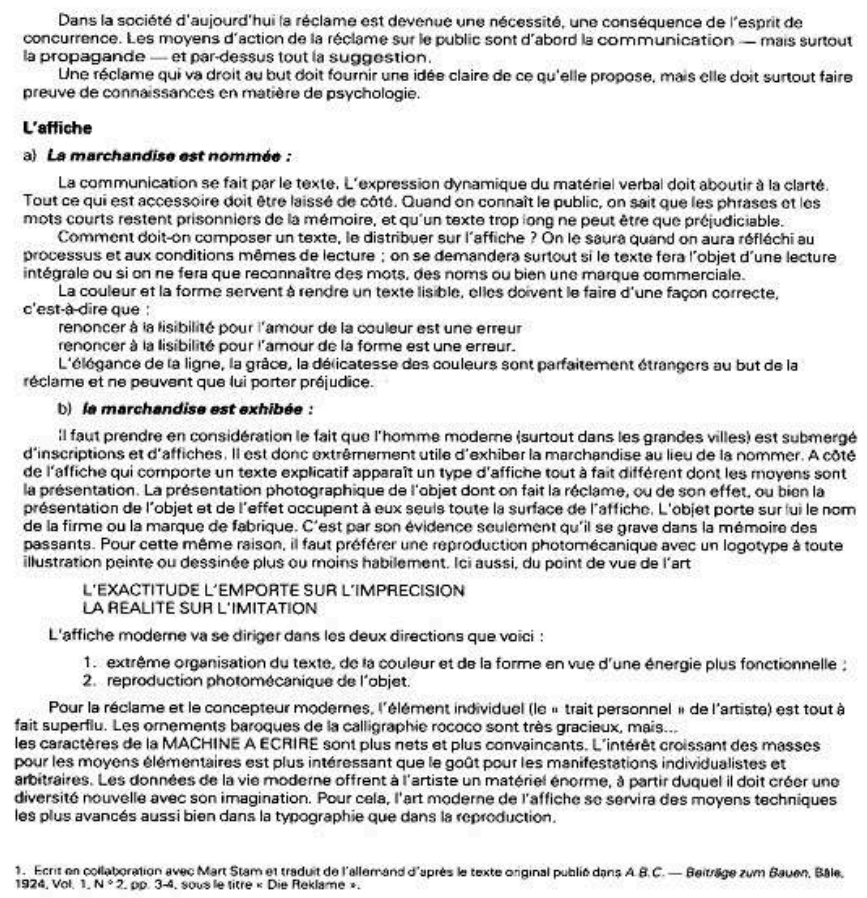

L'espace typographique moderne n'est plus régi par les valeurs classiques d'ordre, de continuité, d'homogénéité et de stabilité mais il obéit à un type d'organisation réglé par les notions de discontinuité, de simultanéité, de contraste du mouvement de l'art moderne. Au jeu typographique, les constructivistes préfèrent le jeu topographique. Le titre Topographie de la typographie choisi par Lissitzky pour l'article publié par Merz en $1923^{18}$ n'est pas simplement un calembour conforme à l'esprit dadaïste de la revue. Le terme topographie, qui désigne la science des lieux et des positions, témoigne que seules l'intéressent, dans un imprimé, les relations des signes typographiques dans l'espace plan matériel - le terrain - que constitue la feuille de papier.

1. Disloquer la séquence phonétique au profit d'une image purement spatiale du mot.

2. Les mots de la surface imprimée sont vus, non pas entendus.

3. Les idées sont communiquées par la convention des mots. Par les lettres, l'idée peut être mise en forme.

4. Economie de l'expression - optique au lieu de phonétique.

5. La conception de l'espace du livre par le matériau de la composition selon les lois de la mécanique typographique doit correspondre aux tensions et aux mouvements du contenu.

6. La conception de l'espace du livre est basée sur le matériau des clichés qui réalisent la nouvelle optique. La réalité supernaturelle de l'œil perfectionné.

61 7. La séquence de pages continue - le livre bioscopique.

62 8. Le nouveau livre demande un nouvel écrivain. L'encrier et la plume sont morts. (volonté révolutionnaire correspondant à l'époque)

63 9. La surface imprimée dépasse l'espace et le temps. La surface imprimée, l'infinité des livres, doivent être dépassées. (l'electro-bibliothèque) 

l'espace classique par la mise en rapport des espaces réputés hétérogènes de la lettre (spatialisée) et de l'image (mise à plat et frontalisée). Comme l'écrit C. Leclanche-Boulé :

«Pour en revenir aux compositions typographiques et aux photomontages, on peut considérer que la lettre, avec un peu plus de sens qu'on ne lui en accorde d'ordinaire et la photographie avec un peu moins de sens qu'il n'y paraît au premier abord, ont un fonctionnement identique à l'intérieur de leur propre espace. A fortiori n'y a-t-il aucun empêchement à les faire fonctionner dans un seul et même espace. ${ }^{19}$

L'œuvre graphique des constructivistes russes dévoilant une portée psychologique et sociale se compose surtout d'affiches et de manifestes ainsi que de revues, rarement de livres dehors des couvertures. Cela s'explique par le faible développement de la polygraphie russe au début du $20^{e}$ siècle. Les constructivistes russes opèrent une mutation de l'espace de la représentation rompant avec la linéarité du discours ou de la représentation perspective. Ils utilisent la géométrie pour découper l'espace de la représentation en une multitude de fragments investis de sens contradictoires. Les photos non détourées, et leur prolifération créent un effet de frontalité et de platitude, la réserve de blanc devient une arme absolue contre le creusement perspectif. Cette structure supprime la hiérarchie de la figure et du fond, du discours et de l'image, de l'entendement et des affects. L'organisation contrastée de la typographie doit servir à renforcer les aspects sensibles avant même de produire du sens. Lissitzky sous-entend que les phénomènes de communication s'effectuent au niveau visuel et non au niveau $\mathrm{du}$ langage. La dimension ludique, le jeu sur des règles arbitraires suspendant les lois ordinaires reste présente. La liberté dans le cadre des règles, la pluralité et l'indétermination du sens associent le lecteur/spectateur à la construction de l'œuvre et invitent le lecteur à faire l'œuvre avec lui, laissant une ouverture intentionnelle du sens. Le thème de l'innervation de la main sur l'œil est récurent chez Lissitzky, il l'illustre radicalement dans l'œuvre suivante :

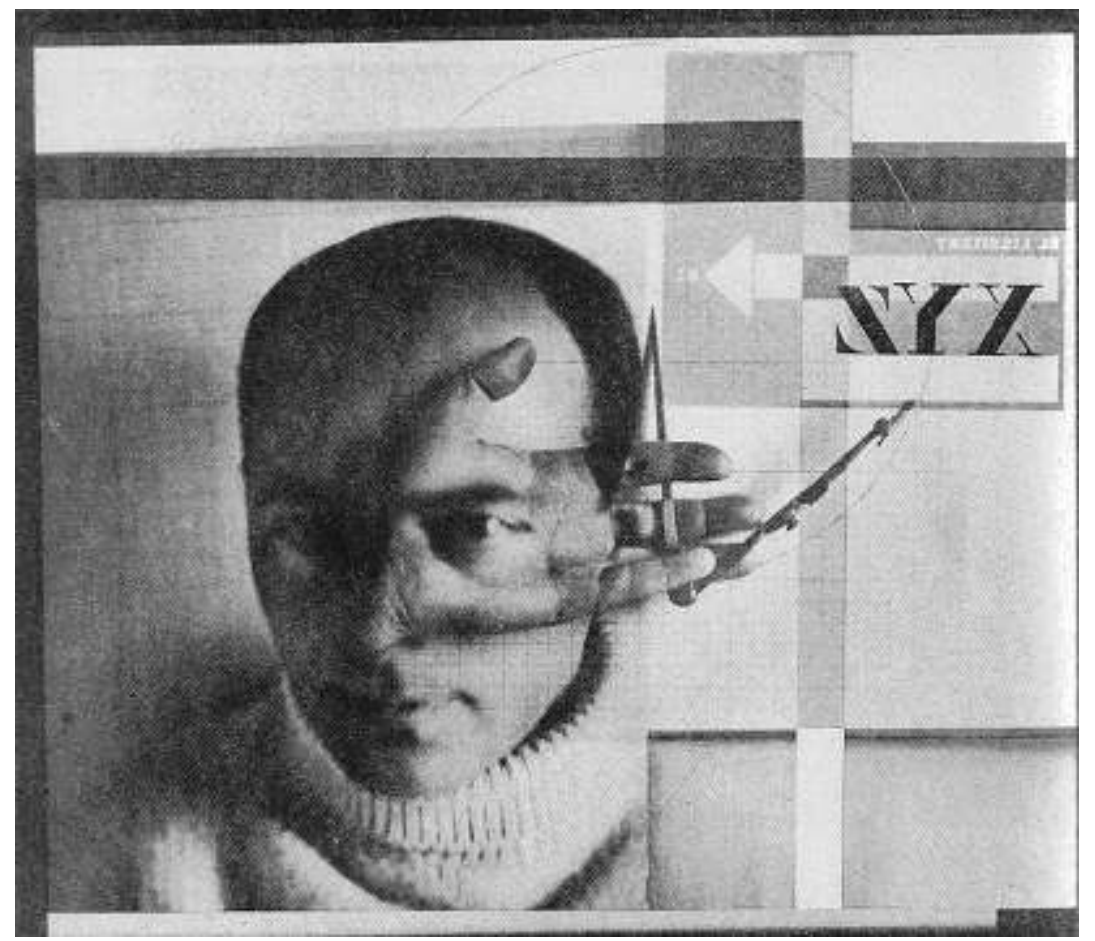

Appareil , Articles 

possible de repartir d'une de ses oeuvres les plus connues :

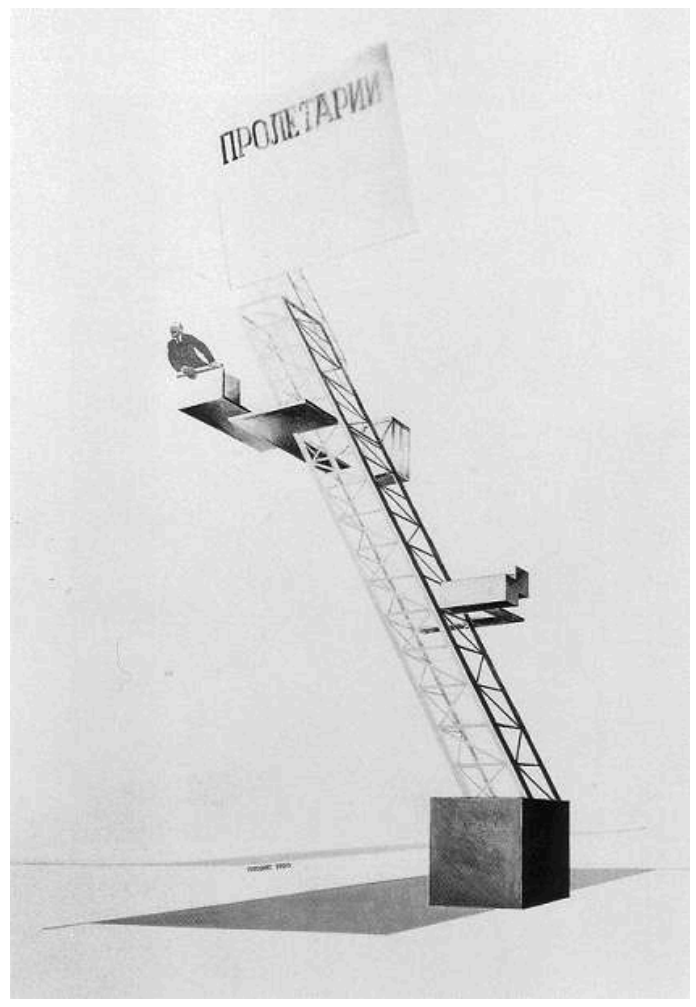

Il utilise dans ce montage une image qui deviendra parmi les plus connues de Lénine.

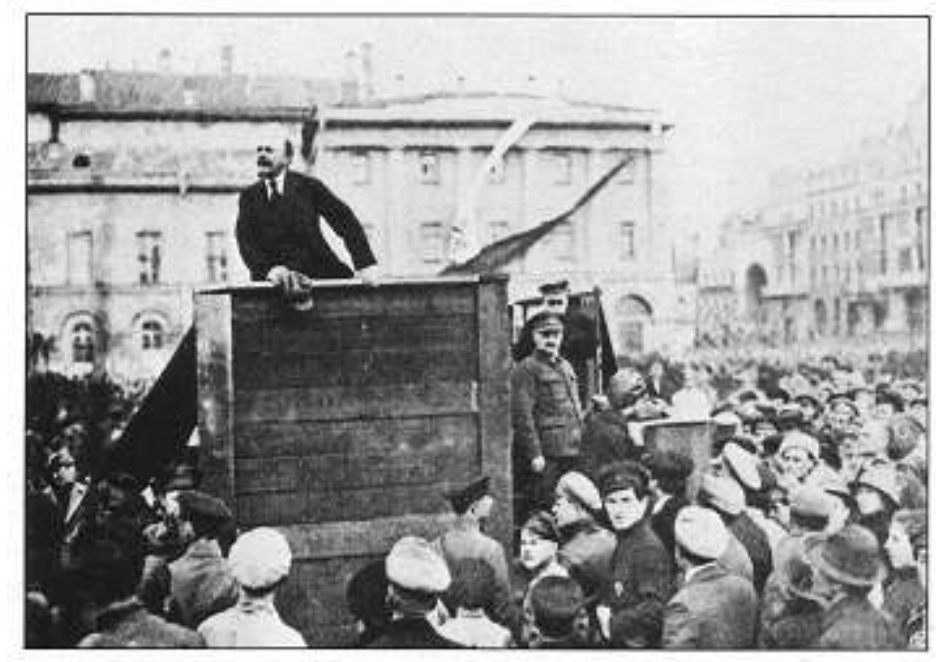

Il est pour moi remarquable que l'artiste transforme un Lénine bien posé sur une tribune massive et terre à terre, légèrement incliné vers une foule qui n'est pas si lointaine, en une figure, certes élevée, mais loin de tous et en complet porte à faux. Compréhension intuitive et anticipée de la réalité de l'avant-garde politique du prolétariat, je ne le pense pas, car à la même époque ou presque Lissitzky fond Lénine dans la foule. 


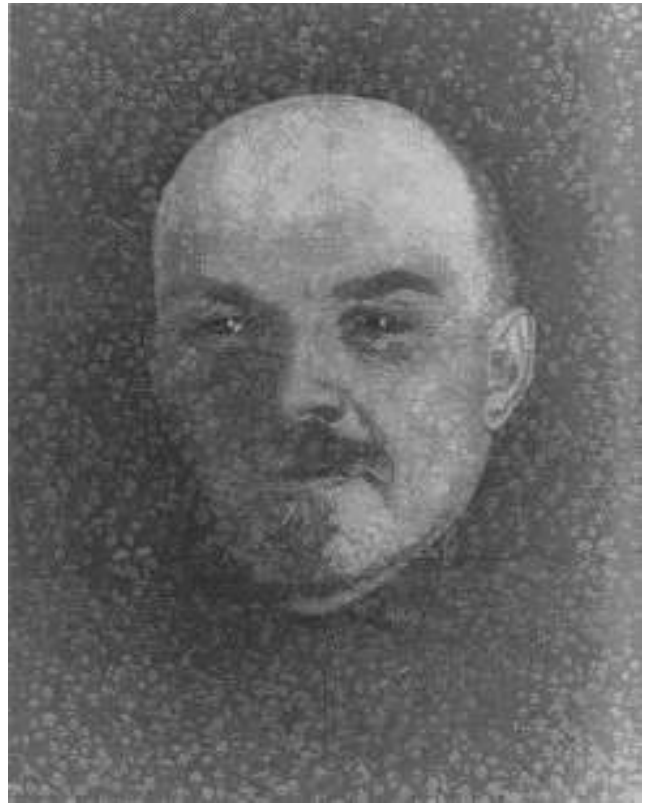

69 Par contre la photo de Lénine à la tribune connaîtra bien des vicissitudes. Sur la photo initiale figurent Trosky et Kaménev. Ils disparaîtront par recadrage ou par retouche pure et simple.
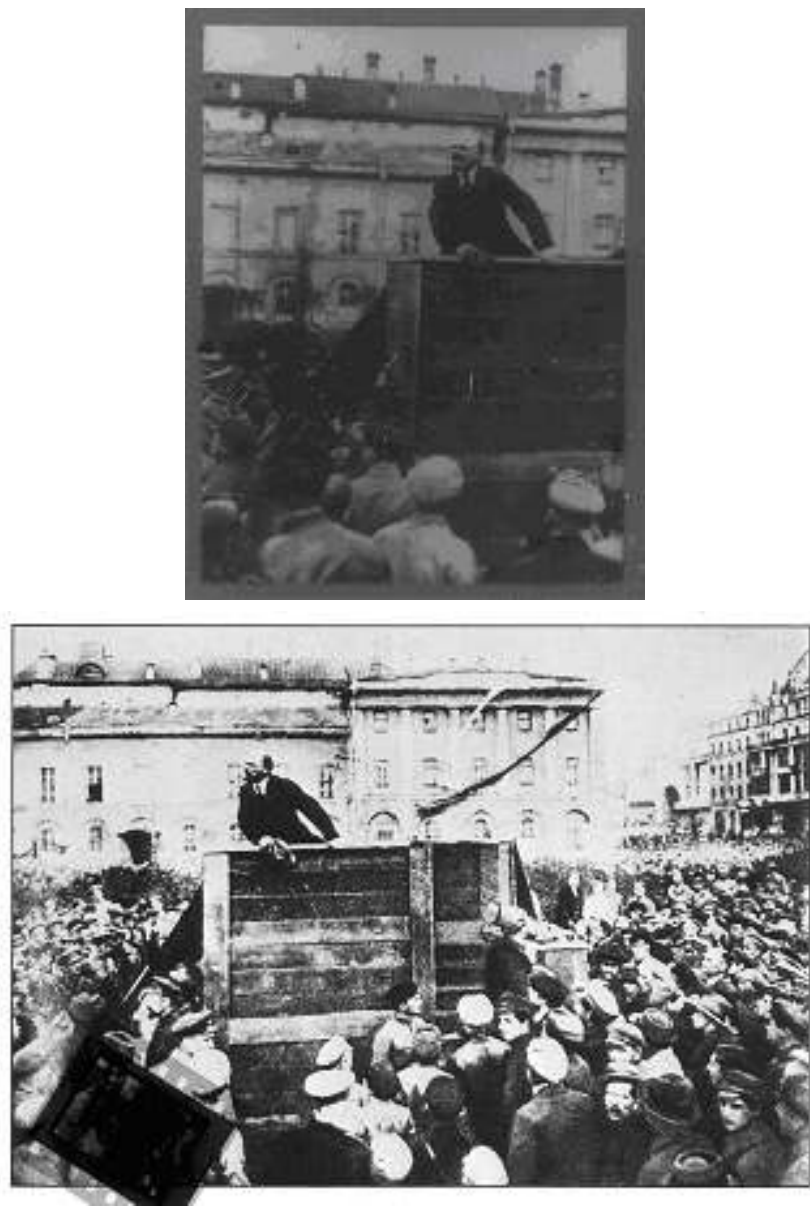

En fait, Lissitzky poursuivra son activité de propagandiste au service de l'URSS au travers de stands, d'affiches et de maquettes pour l'URSS en construction jusqu'à sa mort en 1941 (el-1_stalin). Dans le document L'artiste dans la production, Lissitzky 
s'adresse à un public exclusivement soviétique, en plein essors de la théorie du socialisme dans un seul pays. Soucieux d'éviter des critiques de cosmopolitisme, Lissitzky gomme les influences étrangères sur la typographie, et le photomontage ainsi que les rapports avec les autres pratiques de l'avant-garde. De façon très prudente, il avance que :

«L'attitude des artistes face à la production est très significative. En tout temps, chaque fois que l'artiste a refusé de servir la masse, il a également refusé la "production de masse". Au service des "minces couches supérieures" de la société, l'art est devenu un artisanat, il s'est enfermé dans l'atelier étroit de l'artiste isolé, jusqu'à ce que l'un et l'autre commencent à s'épuiser et s'asphyxier. Avant la Révolution d'Octobre, chez nous aussi, l'art et l'artiste donnaient des signes d'épuisement. En frayant un chemin vers les masses, la Révolution d'octobre a rendu nécessaire le transfert à l'usine et à la machine des expériences menées en privé dans l'atelier et devant le chevalet. De plus, l'œuvre unique, parce qu'elle réclame une énergie hors de proportion avec sa sphère d'influence, est devenue un produit de luxe que les oeuvres imprimées ont supplanté. »

Le régime stalinien continuera à recourir à des artistes comme Lissitzky ou Rodchenko en raison même de la force de cette forme de propagande. Les dernières couvertures de USSR In Bau sont bien loin de l'impact du coin rouge. 

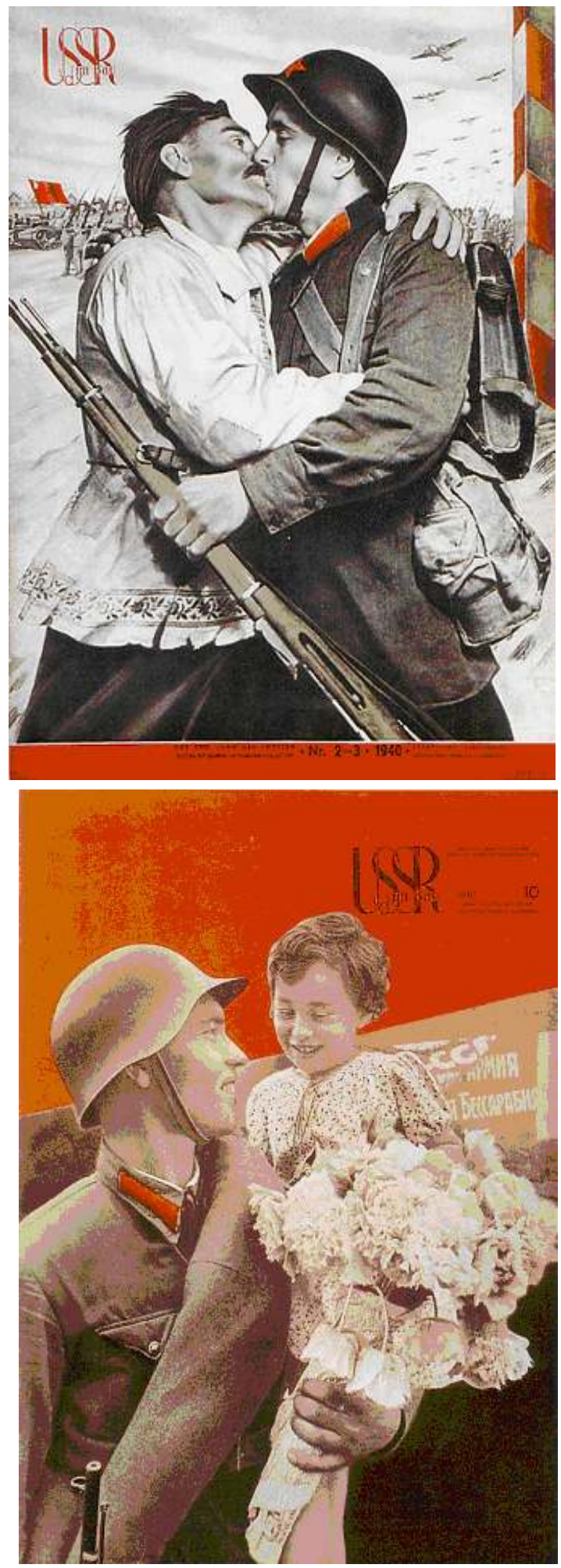

72

Et là aussi la dérive fonctionnaliste est visible même dans cette oeuvre ultime un peu plus inspirée toutefois que les précédentes. Là l'enjeu a fait peut-être que le régime relâche quelque peu la pression. 


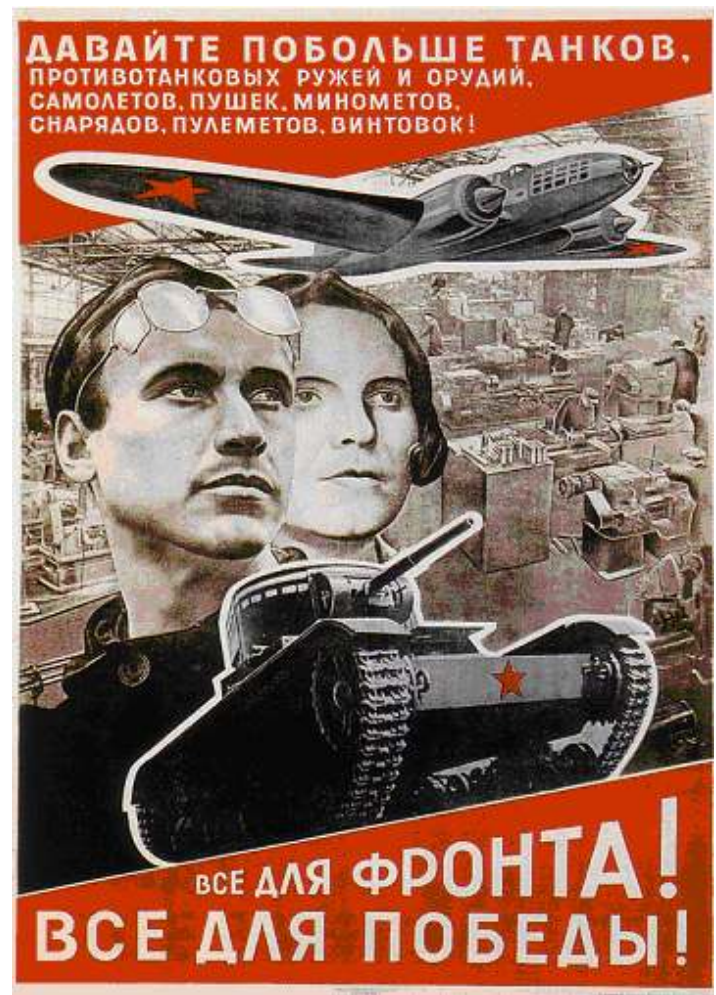

73 Un incontestable mouvement d'émancipation du regard et de la lecture, devenu instrument de la propagande soviétique, inspirera les publicitaires. La veine n'est pas tarie, elle fonctionne encore aujourd'hui.

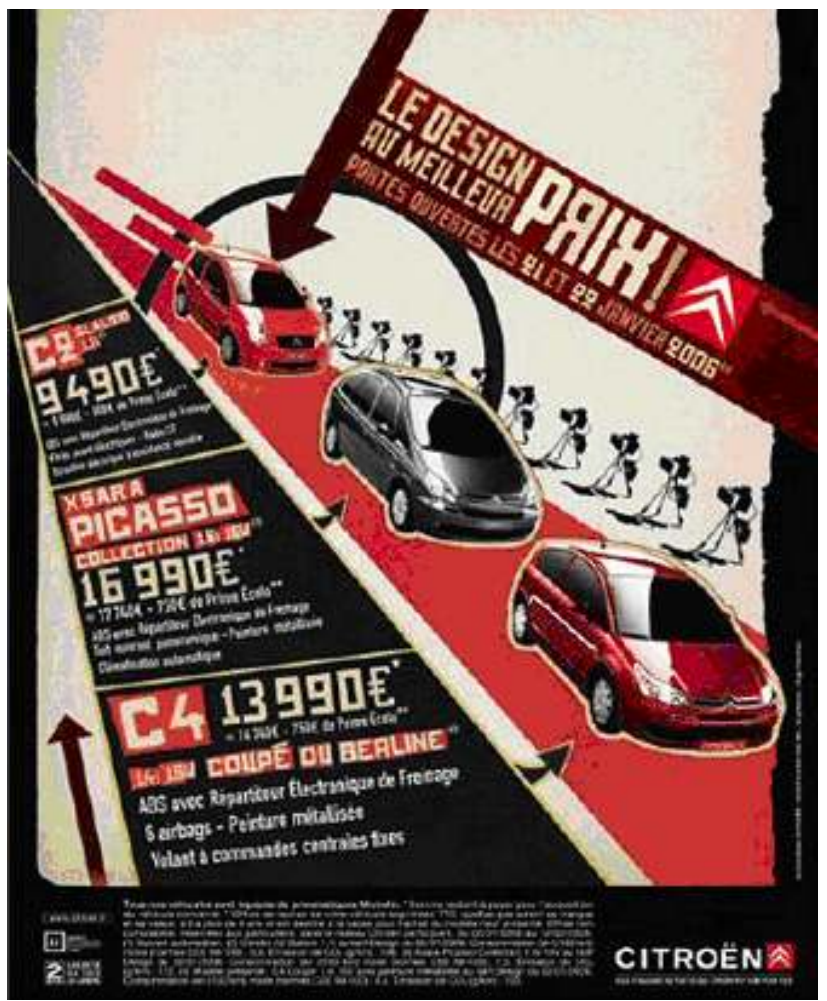

74 Elle influence, parfois de manière inconsciente, mais presque toujours tue, sur de nombreuses productions plastiques contemporaines (souvent par la médiation de 
Brodovitch). En ce qui concerne le groupe musical Kraftwerk, l'influence est limpide et assumée.
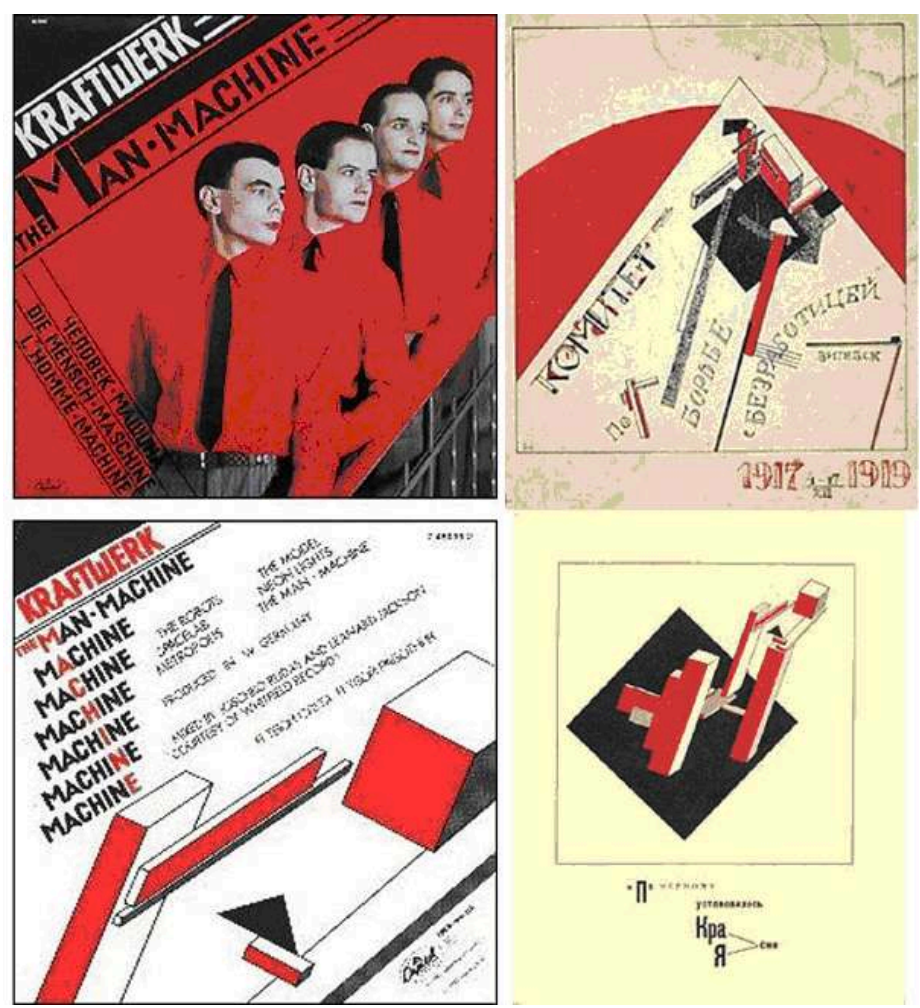

L'étude de la relation des Avant-Gardes au développement des industries culturelles mérite d'être poursuivie. Certaines de ces liaisons sont parfaitement explicite. On peut citer Alexey Brodovitch (directeur artistique de Harper's Bazaar, qui a mis en avant les idées artistiques de l'avant-garde par la médiation d'une revue de mode propriété du très réactionnaire William Randolph Hearst), la naissance du design industriel moderne (avec une réflexion à reprendre sur le Bauhaus, version sociale démocrate du VHUTEINI), l'enjeu central étant du côté du développement de l'ingénierie de contrôle social.

L'analyse faite ici n'est pas une condamnation de l'art des constructivistes. Personne ne songe à exiger de Michel Ange, décorateur de la Chapelle Sixtine, qu'il rende des comptes à propos de l'obscurantisme des Papes. Son objectif est bien plutôt de tenter d'échapper aux sur-simplifications, adorniennes en particulier, de la question des industries culturelles. Si Avec le coin rouge bats les blancs! appartient, par le mouvement que cette oeuvre ouvre, au domaine de l'industrie culturelle, c'est aussi et surtout une très grande oeuvre. 


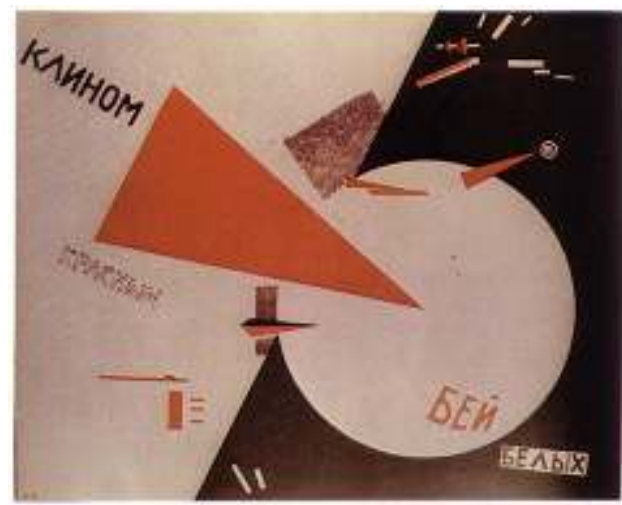

\section{NOTES}

1. Cité par K.P. Zygas : Form Follows Form in Studies in the Fine Art: The Avant-Garde, No 15S.C.Foester UMI research press, AN Arbor Michigan USA, 1978.

2. Konstruktsiia Prostrantrerroye Sooweczkabie ou construction d'un appareil spatial.

3. Margarita Tupitsyn : EL Lissitzky Jenseits der Abstraktion Spregel Museum Hannover, 1999.

4. T. Adorno, Horkenheimer M. : La dialectique de la raison, Tel Gallimard, Paris, 1989.

5. Le dessin est l'objet idéel, il est aussi l'objet idéal, il est aussi, par le tolérancement, l'ensemble des objets jugés acceptables (les autres n'étant que rebuts).

6. M. Porchet in Appareils et formes de la sensibilité J.-L. Déotte (dir.), L'Harmattan, Paris, 2005.

7. B. Arvatov : L'art et la production, recueil d'articles Moscou 1926, cité par M.Zalambani Boris Arvatov, théoricien du productivisme, Cahiers du monde russe, 40/3, juillet-septembre, 1999.

8. Lénine, depuis qu'il avait lu l'ouvrage de Tomaso Campanella, était hanté par une idée qu'il exposa à Lounatcharski : impressionné par la description des fresques à caractère didactique recouvrant les bâtiments de la célèbre cité utopique, Lénine lui avait fait part de sa conception d'une " propagande monumentale " visant à remplacer toutes les anciennes statues des gloires du tsarisme par de nouvelles, dédiées aux grandes figures révolutionnaires.

9. Homme politique, philosophe et médecin, Aleksandr Bogdanov (pseudonyme de Malinovskij) (1873-1928) proclame que la classe ouvrière ne doit en aucun cas subir la contamination des idées de l'intelligencia d'origine bourgeoise. Il est expulsé, en 1909, du parti bolchevique pour avoir polémiqué contre Lénine qui l'attaque rudement dans Matérialisme et empiriocriticisme.

10. V. I. Lénine, PPS, 52, 1965, pp. 179 ; oeuvres, 1970, 5, p. 114.

11. Lounatcharski a séjourné à l'étranger (Zurich, Paris). Il a fréquenté les milieux cubistes et pu se familiarise avec l'art moderne. Il n'est pas membre du Comité Central du Parti Communiste et n'y jouera jamais un rôle important. Il est plutôt reconnu comme un spécialiste au service du Parti et bénéficie de la confiance de Lénine, même s'il s'est parfois opposé à lui. Privé de son poste de Commissaire du peuple, en butte à des humiliations politiques de la part de Staline, il mourra d'une maladie infectieuse le 26 décembre 1933.

12. M. Porchet, "L'appareil numérique et la perspective ou le retour des espaces projectifs en art”, Revue Appareil [en ligne], Articles, Varia, mis à jour le : 17/03/2008, URL :

http://revues.mshparisnord.org/lodel/appareil/index.php?id=303. 
13. Maïakovsky, sept jours d'exposition de la peinture française, cité par C. Leclanche-Boulé : Le constructivisme Russe, Flammarion, Paris, 1991.

14. Le byt, nous dit Arvatov, est le système plus ou moins stable de formes primitives que l'être social assume à chaque instant donné. « La fusion complète des formes artistiques avec celles du byt, la pénétration totale de l'art dans la vie, la création d'une existence sociale parfaitement organisée et rationnelle au plus haut degré, rénovée sans arrêt, rendront la vie harmonieuse, permettront le développement joyeux et complet de toutes les activités sociales et aboliront en outre le concept même de byt. Celui-ci, en effet, implique quelque chose de statique, de raide, qui disparaîtra, étant donné que les formes de l'existence, ce que l'on appelle byt actuellement, se transformeront constamment selon les changements des forces productives. »

15. J.-L. Déotte, Le musée l'origine de l'esthétique, L'Harmattan, 1993.

16. Armin Steil Die imaginäre Revolte. Untersuchungen zur faschistischen Ideologie und ihrer theoretischen Vorbereitung bei Georges Sorel, Carl Schmitt und Ernst Jünger.

17. C. Leclanche-Boulé : Le constructivisme Russe, Flammarion Paris, 1991

18. Cité par C. Leclanche-Boulé : Le constructivisme Russe, Flammarion Paris, 1991

19. C. Leclanche-Boulé : Le constructivisme Russe, Flammarion Paris, 1991

\section{RÉSUMÉS}

La production industrielle, invoquée par les constructivistes, n'est jamais réellement dévoilée, les objets interviennent en termes de propagande ou sont fétichisés, ils ne sont jamais fonctionnels, les machines sont figées et vues de l'extérieur, si le mouvement est suggéré par la forme, il est de fait impossible. Les lois de la physique s'effacent derrière l'enthousiasme productiviste. Ce sont la communication industrielle, le design, les lieux de productions médiatiques (presse, radio, cinéma), les projets de pavillons de foires et expositions qui inspirent et cadrent leurs œuvres. Les industries culturelles pointent le nez. Le développement technique est perçu comme le moteur d'un progrès objectivement émancipateur, source de tous les malentendus qui permettent d'admirer tout à la fois l'industrie américaine et la révolution d'octobre. L'art de la maquette développé par les constructivistes survivra à la condamnation des avant-gardes par le pouvoir soviétique et cœxistera avec le réalisme socialiste de l'art officiel au moins jusqu'à la deuxième guerre mondiale.

\section{INDEX}

Mots-clés : Avant-Garde, conception, constructivisme, dessin technique, fabrication industrielle, fonction, forme, industries culturelles, ingénierie industrielle, ingénierie sociale, organisation scientifique du travail, programme, prolétaire, rationalisme technique, savoir-faire, travailleur, typographie 